\title{
Une femme voyageuse dans les flous artistiques symbolistes: "Devant le miroir," de Marie Krysinska, trio pour vers, prose et vers libre métissé
}

\section{Florence GOULESQUE}

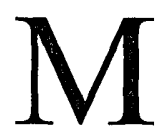

arie Krysinska, femme poète, musicienne, chansonnière et romancière de la période symboliste et décadente fin-de-siècle, quitta sa Pologne natale à l'âge de seize ans pour entrer au Conservatoire National de Musique à Paris. Mais son voyage ne s'arrêta pas là, et son inspiration fuga bien vite hors des murs de l'académie de musique classique, qu'elle quitta bientôt définitivement pour l'atmosphère émancipée et floue des cabarets littéraires et artistiques enfumés. Membre assidu des Hydropathes, puis la seule femme admise aux vendredis littéraires du Chat Noir à Montmartre, elle fut la première, dans les années 80 , à publier des poèmes en vers libres dans La Vie Moderne et autres périodiques de l'époque. Auteur de romans aux titres scandaleux, Folle de son corps (1896 et 1907), et La Force du désir (1905), personnage fougueux et non conventionnel, on l'affubla de tous les noms: "la Sainte Jeanne-Baptistine de l'école verslibriste," "la créatrice du vers libre," "la bonne diablesse," "la verseuse de Chopin" (car elle était pianiste et parfois serveuse au Chat Noir), "le chef du Symbolisme," "la vaillante Polonaise," "la Calliope du Chat Noir," "la diva [adorée] du Chat Noir," "la poémiste en prose," "la Scythe," "la slave," "l'étrangère," et bien d'autres encore. Krysinska s'amusait des chansons qu'elle inspira, telle celle de F. A. Cazals qui donnait l'ordre de: 
Laissez passer cette cris' là

La crise à Krysinska

... ce cri-là

C'est le cri d'Krysinska.

Mais comment cette femme audacieuse, sujette aux plaisanteries des groupes à majorité masculine qu'elle fréquentait, se voyait-elle elle-même? Dans le poème "Devant le miroir," (Joies Errantes 35) une femme se regarde, et s'interroge au long d'un voyage à l'intérieur d'elle-même, qui l'entraîne ensuite vers l'Idéal:




Ce poème traduit la difficulté pour une femme, à la fin du dix-neuvième siècle, de se reconnaître dans l'image que lui renvoie le miroir, dans l'image de la féminité que la société française lui impose, et dans l'ambiguïté de sa position d'exilée et d'étrangère. Le miroir de l'immigrée a aussi trois faces, et elle erre dans le périmètre de ce triangle, ou joue un trio pour la voix de la Polonaise, celle de la Française, mais aussi la voix de quelqu'un d'autre entre les deux, de mal défini. Ce qui est mal défini fait peur, et, à l'époque, parallèlement à une vague de popularité en faveur d'un cosmopolitisme littéraire, sévissait aussi une méfiance vis-à-vis de l'étranger.

Ses origines étrangères ont sans doute aidé Krysinska à se débarrasser du rythme hallucinant de l'alexandrin, par exemple, qu'elle n'avait pas "dans l'oreille," contrairement à beaucoup de Français, et à créer des rythmes poétiques nouveaux. Mais nous pouvons remarquer que, dans son œuvre, presque toutes ses références culturelles sont françaises, et les traces de la culture polonaise ont été comme volontairement effacées. Pour tenter d'être acceptée plus ou moins par la culture française, il a fallu qu'elle négocie son identité, qu'elle abandonne son origine. Krysinska ne s'est pas associée aux nombreux immigrés polonais à Paris à la charnière du siècle, et n'a pas chanté le patriotisme envers la nation menacée, comme cela était typique des écrivains polonais immigrés, ou même chez une contemporaine comme Marie Nizet. Celle-ci, qui pourtant n'avait jamais vu la Roumanie de son père, voyagea dans l'histoire, l'espace slave, et son imagination, et se créa un passé et une identité allant au-delà des limites de sa propre existence spacio-temporelle. Mais Krysinska n'a pas écrit ce manque, l'absence du pays d'origine à proprement parler. L'héritage polonais surgit de l'inconscient avec la danse, les chansons, les rythmes musicaux et le goût du surnaturel, comme par l'effet de la mémoire involontaire de Proust avant la lettre, souvent par l'intermédiaire d'un parfum ou d'un air de musique. De même, Krysinska a cherché à exploiter les effets expressifs de la dissonance plutôt que de décrire explicitement ses sentiments par rapport à l'exil, et sa situation de marginale, dissonante par rapport à une culture dominante française. C'est donc une écriture mouvante qui reflète l'errance de l'exilée.

De même, dans "Devant le miroir," cette errance de l'exilée est perceptible dans la structure de ce poème, plutôt que dans ses thèmes. Il y a un balancement entre les verbes de mouvement, comme "aller," "venir," "commencer," "tomber," "pousser," etc . . . et les verbes ou expressions impersonnelles, comme "il se peut," "on dirait," etc ... Cette alternance est symbolique de la difficulté de l'exilée de fixer son identité: elle est soit errante, en mouvement, comme le suggère aussi le titre du recueil d'où est tiré ce poème, Joies errantes, soit dépersonnalisée car sujette à la définition 
des membres de la culture dominante, qui, motivés par leur peur de "l'autre," tentent d'anéantir sa spécificité. De cette instabilité de l'identité, naît une œuvre mouvante, hybride, caractérisée par un métissage de langues, le mot métissage étant pris ici sans connotations raciales, mais plutôt dans son sens étymologique de mélange de fibres différentes dans l'élaboration d'un tissu. Les fibres de ce poème sont tissées de telle manière que les motifs imprimés en deviennent des images réfléchies dans un miroir, qui est aussi bien sûr le thème du poème. La forme de ce poème en vers libres épouse le fond, puisque sa structure est caractérisée par des jeux de miroir et des relations triangulaires.

Dans la deuxième strophe, des triangles se répondent entre "aller," "vient" et "n'être pas," et dans la dernière strophe, entre "songe," "tombé" et "poussaient." Ces verbes indiquent un même mouvement contradictoire (aller et venir, tomber et pousser) qui déstabilise la réalité de l'être, puisqu'il n'existe plus ("n'être pas"), ou seulement en rêve ("songe"). Le triangle des trois verbes introduits par un "on" dépersonnalisant du sujet, "on voudrait," "on dirait" et "on croyait," fait miroir à celui des trois autres expressions impersonnelles séparées par des tirets "est-ce bien soi," "il se peut," et "en somme," qui suggèrent des interrogations ou des suppositions, et expriment le doute. Enfin, entre les mots "apparue" de la première strophe, et "retrouve" et "disparus" (et son écho au vers précédent, "révolus") de la quatrième strophe, se dessine un triangle qui lui-même est un reflet agrandi de celui de la première strophe entre les mots "entrevue," "connue," et "apparue." Ces deux triangles expriment les conditions du mystère, du mystère que l'on est pour soi-même et du mystère des choses autour de soi: on peut entrevoir ("entrevue") le mystère (mouvement conscient, qui dépend de la volonté du sujet), il peut apparaître ("apparue") (mouvement inconscient, qui ne dépend pas de la volonté du sujet), mais le connaître ou le retrouver ("connue," "retrouve") est une illusion, ou une impression fugitive.

Le monde de Krysinska est en mouvement, et les choses, de même que l'image physique de soi, ne sont qu'illusions, qui ne font qu'apparaître et disparaître. Comme le triangle a trois côtés, le miroir devant lequel s'interroge la femme poète exilée a trois faces: ce qu'elle désire être, l'image que les autres lui disent d'être, et ce qu'elle est vraiment, qui restera toujours un tant soit peu mystérieux. Le marginal, nomade, exilé, artiste, s'il ou elle est mystère, changeant et déroutant pour les autres, l'est aussi pour lui-même ou elle-même.

Mais c'est aussi son ambiguité qui fait sa force, puisqu'en restant dans le flou, Krysinska a échappé à toute catégorisation, et s'est créée (de façon existentialiste avant la lettre) pionnière d'une nouvelle forme poétique 
entre les vers et le poème en prose. Son indépendance et son originalité ont ennuyé bon nombre d'autres poètes et de critiques, férus de catégorisations et d'ordre. Mais Krysinska a toujours tenu bon dans sa revendication de fidélité à elle-même dans toutes ses facettes. Ainsi, dans la première strophe de "Devant le miroir," la rencontre avec l'image de soimême dans le miroir est à la fois "grave" et "fertile en émois." Toutes les parties d'elle-même sont sommées dans cet instant à la fois de vanité et d'introspection: son esprit, avec la lucidité et la capacité de raisonnement, aussi bien que son cœur ou ses émotions, et bien sûr son corps puisque c'est cela qu'elle regarde dans le miroir. Cette revendication d'une intellectuelle sensuelle manifeste un premier niveau de métissage qui empêche une catégorisation stricte dans un domaine ou l'autre, l'esprit ou les sens. Les deux ne sont pas incompatibles, au contraire, dans la poésie de Krysinska, la danse du corps est indissociable de la liberté de l'esprit. Ce métissage des sens, des émotions et de l'intellect est subversif de l'image de la femme écrivain au dix-neuvième siècle, que l'on a souvent voulu catégoriser soit comme un bas-bleu sans sensualité ni sexualité, soit comme une oisive ne cherchant avec la poésie qu'à exprimer sa sensibilité dans l'amour, la nature et la maternité. Krysinska propose une troisième possibilité: un métissage entre l'esprit et le corps, la raison et le cœur.

Le mot "image" suggère non seulement l'image physique dans le miroir, mais aussi l'image poétique. L'intellect, les émotions et les sens participent à la rencontre avec soi-même ainsi qu'à la création poétique. Le premier vers présente le travail poétique comme très sérieux ("grave"), ce qui est confirmé par les écrits théoriques de Marie Krysinska. Il était important pour Krysinska de prouver que sa poésie était le résultat d'un travail théorique, afin de se défendre contre des accusations de facilité, critiques d'ailleurs dirigées contre tous les auteurs de vers libres de l'époque, telle celle d'André Barre, qui attribua l'aspect non fini des poèmes de Krysinska à la paresse "typiquement slave et féminine" (335). Krysinska a donc expliqué, dans les introductions à ses œuvres, les techniques de son travail qui ne se voulait pas du tout anarchique, mais répondait au contraire à des règles précises. Elle présenta ainsi certains de ses procédés:

Nos libertés consistent: à rimer ou à assonancer pour l'oreille seule (sans souci des singuliers et des pluriels) des vers qui se meuvent en des coupes alternantes, réservant, au contraire, l'effet d'une rime riche ou d'un alexandrin pour une chute de strophe qui se boucle ainsi comme une souple draperie sur un joyau d'or- 
sans que l'effet en soit escompté par la monotonie du procédé. (Intermèdes xxviii)

Mais, s'il est produit d'un travail objectif, le poème en vers libres est aussi reflet d'une subjectivité, et "fertile en émois." L'écriture se nourrit d'ellemême et des émotions du poète qui laisse le flot de sa conscience s'exprimer. L'écriture a aussi un effet sur le poète en train d'écrire, puisqu'elle provoque en lui des "émois," mot qui appartient aussi au vocabulaire amoureux. Ces "émois" sont donc les signes qu'un rapport affectif ou passionné s'établit entre le poète et l'acte d'écrire, et que l'entreprise de l'écriture est aussi une aventure exaltante.

Mais ce départ est hésitant, car on ne sait pas vraiment qui l'on est et ce qui nous attend, et la première strophe de "Devant le miroir" se termine par un point d'interrogation angoissé. Les adjectifs "entrevue," "connue," et "apparue" se répondent, comme les étapes de cette découverte difficile de soi-même. On ne peut seulement qu'entrevoir son identité, elle ne peut seulement qu'apparaître soudainement et furtivement, tandis que l'on croit seulement la connaître, mais on ne la reconnaît pas, elle "surprend toujours." De même, selon l'esthétique et la philosophie des Symbolistes, on ne peut qu'entrevoir la vérité qui se cache derrière les choses matérielles que l'on croit connaître, mais dont l'aspect mystérieux "surprend toujours." Comme l'image de soi-même paraît étrange et inattendue, l'image poétique surgit dans l'esprit du poète à son insu, et elle doit aussi surprendre le lecteur. Ainsi, pour Krysinska:

Notre proposition d'art est celle-ci: atteindre au plus de Beauté expressive possible, par le moyen lyrique, subordonnant le cadre aux exigences imprévues de l'image, et rechercher assidûment la surprise de style comme dans la libre prose avec, de plus, le souci d'un rythme particulier qui doit déterminer le caractère poétique déjà établi par le ton ou pour mieux dire le diapason ÉLEVÉ du langage. (Joies errantes vi)

La surprise est telle qu'elle suspend la parole pendant quelques secondes, ou coupe le souffle, ce qui est exprimé par le tiret devant l'expression "-est-ce bien soi." Cet étonnement est en partie celui de l'étrangère qui est aussi étrangère à elle-même. Si l'ambiguïté de l'identité permet le renouvellement de soi, elle est aussi marquée par une division de la conscience: l'observatrice interroge l'observée avec une anxiété qui empêche la prise de parole à la première personne et l'image n'est plus 
alors qu'une troisième personne ("est-ce bien soi" et non pas "est-ce bien moi"), qui s'éloigne encore plus du sujet quand elle est caractérisée comme "cette soudaine apparue," moins qu'une personne, une vision, peut-être une revenante. L'aspect vaporeux de cette vision soudaine évoque aussi le flou artistique, descendant direct de la peinture impressionniste, et utilisé en photographie pour adoucir ou embellir les traits d'un portrait.

De même, Krysinska a favorisé dans son œuvre des thèmes évocateurs de mondes flous, ce qui figure un refus de s'ancrer dans la réalité, et correspond à une volonté de ne pas fixer le texte, ni le moi. Elle a exprimé une prédilection pour les apparitions et disparitions de "Mirages," les portraits de femmes à contre-jour d'“Ombres féminines," fantômes de femmes errantes préfigurant la "présence d'absence" de Paul Valéry, les échos de "Guitares lointaines," les esquisses de "Sketch Book," les demiteintes d" "Automnales," les lumières atténuées de "Clairs de soleil et clairs de lune," les moments incertains d'“Aurores" et de "Crépuscules," où les contours trop bien définis et trop blessants des choses s'estompent, et qui apportent le soulagement et la paix. Tous ces mondes flous sont des répondants poétiques de l'art impressionniste, et, le regard voilé dans le rêve ou la méditation, protégée par l'anonymat de l'ombre, la femme poète errante se cherche une âme, ou poursuit l'Idéal, quelque chose de constant en dehors du temps et de l'espace, et en dehors des normes de la société. Ce refus de se définir dans une réalité concrète correspond donc à l'ambition symboliste de découvrir et d'exprimer un autre monde au-delà des apparences matérielles, mais aussi signifie le désir de ne pas limiter le moi, et d'échapper ainsi aux contraintes de rôles préfabriqués imposés à la femme en particulier.

Parallèlement à cette volonté de rester dans le vague, Krysinska n'a utilisé le "je" que dans quelques poèmes isolés parmi les deux cent poèmes de ses trois recueils. Ses romans et ses nouvelles ne sont pas écrits non plus à la première personne. Krysinska a une prédilection pour le "nous" et le "on," et pour raconter les autres, à travers lesquels elle se révèle sans doute, mais aussi derrière lesquels elle se cache. L'hésitation à utiliser le "je" peut illustrer la difficulté pour la femme de ce qu'Hélène Cixous a appelé "la venue à l'écriture," le long et pénible chemin de l'image/objet désirant devenir sujet écrivant une expérience authentique. En effet, comme le montre la deuxième strophe de "Devant le miroir," si la reconnaissance de l'image de soi-même pose problème, c'est parce qu'elle est définie par les autres. Une tentative est faite ici pour adhérer à l'image de la féminité imposée à la femme par les codes socio-culturels de l'époque, une image de superficialité, de vanité et de puérilité représentée par "les petites mines." Il y a une tension entre ce que l'on voudrait être et ce que les autres 
voudraient que l'on soit. On semble adhérer tant soit peu à l'image conventionnelle de la femme, afin de combattre le risque de non existence qui menace, comme l'exprime le vers, "l'inquiétude / de n'être pas-il se peut." Ce risque est réel: le tiret et l'expression "il se peut" isolent et mettent en relief le "n'être pas," l'anéantissement de l'être, possible si l'image n'est pas bonne ou conforme, et que l'on ne sait pas quoi mettre à la place. Il semble qu'il n'y ait donc que deux possibilités pour la femme: la conformité aux modèles féminins dominants, ou la non existence. La première possibilité, faire des mines et être "aussi bien qu'on voudrait," n'étant d'ailleurs pas plus avenante que la deuxième, puisqu'une partie de soi-même est aliénée, le "soi" de la première strophe étant devenu un "on" plus anonyme, qui est à la fois une représentation de son identité encore plus éloignée de soi-même, et plus proche de la masse des autres.

Mais, entre ces deux possibilités, tout autant insatisfaisantes l'une que l'autre, Krysinska en offre une troisième: c'est celle de l'ambiguïté, une fois encore, rester dans le flou. En effet, l'expression "on voudrait" peut signifier "ce que les autres voudraient," mais aussi ce que "je voudrais." Ce n'est pas clair, et c'est en se cachant dans l'ombre, ou dans les doubles sens, que le poète se crée inattaquable, et c'est en donnant la parole aux autres-apparemment-qu'elle se met en position de force. Cette troisième possibilité, l'ambiguïté, est évocateur du troisième côté du triangle, et déconstructif des oppositions binaires. Krysinska ruse et joue avec l'image traditionnelle de la féminité, en la ridiculisant, par exemple avec l'expression saugrenue "les petites mines," afin de mieux la subvertir. Elle revêt ellemême le masque d'une féminité superficielle, pour s'accorder le droit, sous le masque, d'être différente et mystérieuse.

De même, c'est en jouant avec le langage même, qu'elle le transforme. En ce qui concerne l'écriture, on remarque aussi le même schéma triangulaire que pour la féminité: d'abord un balancement entre deux pôles, l'apparente conformité-aux formes poétiques traditionnelles-et le risque de non existence-la marginalisation du poème-, puis la résolution de ce dilemne par une ambiguité prosodique subversive. En effet, le risque est d'abord réel pour le poème d'être considéré comme mauvais, et d'être rejeté s'il n'adhère pas aux normes littéraires de son temps, angoisse de l'artiste d'avant-garde, voulant à la fois être différente et acceptée. La forme même du poème suggère cette tension. Il y a d'une part le désir de se créer soi-même, unique et libre, représenté par le travail du vers libre, avec des vers de longueurs différentes et aux rythmes variés, ainsi qu'une organisation en strophes inégales et une typographie dansante. Et, d'autre part, il y a la nécessité de se conformer à une image traditionnelle, suggérée par les rimes, inhabituelles dans la poésie de Krysinska, avec une 
abondance ici de voyelles claires, ou aigües, elles-mêmes évocatrices de cette tension, de ce cri intérieur. Les "petites mines" pourraient alors représenter les rimes ou autres procédés poétiques conventionnels auxquels le poète pourrait se rattacher pour essayer d'être "aussi bien qu'on voudrait," le "on" pouvant ici représenter les académiciens déterminant le canon littéraire, ou bien seulement le public français non habitué à la forme en vers libres de l'étrangère Krysinska. L'homophonie entre "mines" et "rimes" suggère cette analogie, mais aussi ce que Krysinska elle-même pensait des rimeurs:

Aussi, voit-on une avalanche de médiocres auteurs en vers s'embarrassant dans des superfluités que motive le métrage obligatoire, sombrant dans le galimatiasgrâce à des difficultés mal vaincues-parlant petit nègre, réduits à cette extrêmité par la tyranie de la rime, la suzeraineté de la césure, et les intransigeances de la consonne d'appui. (Intermèdes xxii)

Cependant, les rimes de "Devant le miroir" ne suivent pas les lois classiques, puisqu'elles peuvent se schématiser ainsi: $\mathrm{a} b \mathrm{a} \mathrm{b}$ a / $\mathrm{c} \mathrm{d} \mathrm{d} \mathrm{c} \mathrm{/}$ e e c c / c a a a / c f c c f. Entre la conformité des rimes, et leur absence, Krysinska offre donc une troisième possibilité, des rimes fantaisistes, des rimes qui n'en sont pas, un genre poétique nouveau et trouble. Krysinska, "qui fait dans les coins des duos de chahut [avec Mallet de Bassilau aux Hydropathes]" (cité dans Michaud 229), voyage dans le paradoxe, elle subvertit tout ce qu'elle touche avec un air de se conformer. C'est le mouvement qui l'encourage à prendre des libertés. Ici, les verbes "aller," "venir" et "n'être pas" suggèrent la situation triangulaire de l'immigrante, qui va, qui vient, qui peut se perdre dans ce va-et-vient, mais qui reste insaisissable. Elle est nomade, errante, et a du mal à se fixer quelque part, sinon physiquement, du moins dans sa représentation d'elle-même, si bien qu'elle a parfois le sentiment de "n'être pas," mais elle ne dit pas "je ne suis pas." Elle "est," mais ailleurs, dans un espace indéfini.

De cette "présence d'absence" (Paul Valéry) vient, dans les troisième et quatrième strophes du poème, "une distribution de récompenses généreuses," qui comporte aussi un risque: la perte de l'intégrité de la personne. D'une division en deux, observatrice/observée, identité/image, sujet/objet, conformité/non existence, le moi a découvert une troisième possibilité, l'ambiguïté, qui conduit à l'éclatement en mille morceaux, dont, par exemple, les cheveux, qui sont eux-mêmes un groupe de milliers d'éléments distincts. Mais les cheveux sont surtout parfaits vus de profil 
("Surtout de profil"), ce qui est en quelque sorte la position de l'immigré. Le marginal est à l'extérieur de la société et regarde donc les choses de côté, et non en face, ni de l'intérieur. Sa voix en apparaît dissonante. Les rythmes dissonants de ce poème, une impression de cahin-caha, expriment aussi cette dislocation. Le langage lui-même s'en trouve désarticulé et cette désagrégation apparaît par un métissage de tons: le langage poétique devient langage parlé et ironique ("Les cheveux? ah! les cheveux, parfait!"). A nouveau, c'est dans les jeux de langage que Krysinska offre une possibilité d'être fidèle à elle-même et à ses dissonances. Krysinska a beaucoup insisté dans ses écrits théoriques sur ce désir de dissonance, qu'elle élève en principe esthétique, comme l'ensemble des "diversions et brisements de la rythmique, en vue de l'effet précieux d'inattendu et de rupture de monotonie" (Intermèdes xvii). Attentive à la musique changeante de son âme et fidèle à tous ses désordres, elle "a peint, non la chose, mais l'effet qu'elle produit," selon la formule de Mallarmé (cité dans Godfrey 720 ), et selon le désir des peintres impressionnistes de créer une sensation.

Ces "récompenses généreuses" peuvent aussi représenter les joies rencontrées lors du processus de création artistique, à la fois source de plaisir et de souffrance, libération et travail, ce qui renvoie à "émois" et "grave" de la première strophe. Le poète souffre de cette dislocation, tout en la sachant nécessaire, tout comme Paul Valéry montrera dans "La Jeune Parque" la division de la conscience en train de créer. Cette dislocation est peut-être aussi une condition pour mieux se retrouver. Alors on recherche un état antérieur à cette dislocation, et il faut remonter au commencement de la vie ("commence"), à la naissance, ou au début de l'enfance, où l'on espère trouver un être unifié.

Ce voyage vers l'enfance prend du temps, de même que la réflexion sur soi-même et l'écriture ne suivent pas des chemins uniformes dans le temps. Il y a parfois des hésitations, signifiées par exemple par la coupure de la phrase "on dirait / De telle peinture d'artiste admiré" entre les troisième et quatrième strophes du poème. Le blanc sur la page est important, car il suggère une interrogation, la difficulté de se comprendre, ou celle d'écrire. Dans la quatrième strophe, l'image changeante s'est transformée en "peinture," un portrait figé peint par quelqu'un d'autre, "un artiste admiré," c'est-à-dire, un homme ("admiré" est masculin), et un artiste reconnu par le canon, puisqu'il est admiré. C'est donc la culture dominante masculine qui tente de peindre l'image de la culture marginale féminine. La femme ne bouge plus dans le portrait, elle est comme l'autre la voit, un bel objet. Cependant, une fois l'image immobilisée, on peut y observer les "menus 
détails" qui sont les traits de l'enfance, tout comme une forme poétique fixe traditionnelle peut être découpée et manipulée, et l'attention portée aux "menus détails," c'est à dire aux mots eux-mêmes. Que veulent vraiment dire les mots et comment peut-on manipuler le langage pour le faire sien? Ces questions sont celles que se sont posées les verslibristes et Marie Krysinska.

A nouveau, Krysinska ruse, elle utilise cet immobilisme et cette dislocation mêmes, imposées de l'extérieur, pour s'en libérer. En effet, à partir de la stabilité et de la fragmentation, on peut se connaître, observer, dans les "détails menus," ses différents "moi" comme le fait le narrateur de Proust dans $A$ la Recherche du temps perdu, et créer du nouveau. Ces signes aperçus dans les morceaux de l'image brisée peuvent aussi prendre force par un effet de multiplication, les variations à l'infini d'une personnalité multiple, riche, insaisissable, comme dans le kaléidoscope du "Cœur innombrable" d'Anna de Noailles (1876-1933). L'image, immobilisée pour un instant dans le portrait fait par un "autre," est certes disloquée, mais cette division finit par produire une transformation. En effet, dans ce miroir désormais brisé, comme le poème est brisé en strophes inégales et en rythmes variés, ce n'est pas le moi présent que l'on découvre dans chaque parcelle isolée, dans les "détails menus" de son visage, mais le moi des “jours enfantins." L'enfance est le pays d'origine, ce qui a formé le poète, car, comme l'a exprimé Lucie Delarue-Mardrus (1880-1945): “Ah! je ne guérirai jamais de mon pays! / Et qui donc a jamais guéri de son enfance?" ("L'Odeur de mon pays"). Mais ce moi des "jours enfantins" est ici très fugitif, et, aussitôt qu'il apparaît, il disparaît ("révolus," "disparus"). Rien n'est stable, et le retour à l'enfance n'est qu'une étape nécessaire vers une transformation ultérieure. Une liberté absolue réclame un détachement de tout, même, et peut-être surtout de l'enfance, aussi rassurant que puisse être cet espace. Le désir de rester insaisissable est reflété dans un texte où rien n'est définitif. Les choses auxquelles nous tenons le plus sont les choses qui passent, et elles prennent leur force de leur évanescence même.

Car, encore une fois, c'est cette ambiguïté, ce mystère, cette image/ mirage qui permet de créer quelque chose de tout à fait original. Richard Stamelman commente le poème en prose "L'Etranger" de Baudelaire, en affirmant que l'étranger se libère parce qu'il est l'autre et détaché de tout (118-9). Pour le bourgeois qui interroge "l'énigmatique étranger" bohème, ce dernier n'a rien, il vit dans un monde de manque, sans forme et sans frontières. Il est libre, insaisissable, et il ne peut être fait objet. La seule chose qu'il possède c'est une langue bien à lui, un langage poétique réfléchissant lui-même l'insaisissable: "les merveilleux nuages." A l'exil 
par rapport à une forme de vie conventionnelle, répond donc une libération de l'expression poétique. Ainsi Krysinska, avec le vers libre, les rythmes irréguliers, la dissonance, l'assymétrie, les images surprenantes et le déséquilibre de la syntaxe, s'est créée sa propre langue, libérée des formes traditionnelles, et insaisissable. La marginale risque l'oubli, certes, mais elle est aussi libre d'innover totalement.

Krysinska représente une quadruple marginalisation et altérité: celles de la femme, objet privé de langue, et séparée de son corps, celles de l'étrangère, dépourvue de stabilité, celles du poète ou de l'écrivain, qui s'isolent pour créer, et celles du poète symboliste ou décadent plus ou moins anarchique et bohème, exilé du monde positiviste. A cela on peut ajouter que Krysinska elle-même refusa d'appartenir à aucun mouvement, même s'il était d'avant-garde, et que certains poètes symbolistes et décadents la rejetèrent quand ils refusèrent de reconnaître son rôle actif parmi les efforts innovateurs de la forme poétique libre, et quand ils critiquèrent son abus de clarté dans sa poésie.

Elle ne put non plus s'associer aux autres femmes poètes de la fin du dix-neuvième siècle ou du début du vingtième siècle, parmi lesquelles Anna de Noailles, Hélène Vacaresco, Marguerite Burnat-Provins, Marie Nizet, etc ..., étaient aussi d'origine étrangère. L'errance de ces dernières a contribué à la libération de l'expression poétique de leur sexualité avec, d'une part, le lesbianisme chez Renée Vivien et Nathalie Clifford-Barney, et d'autre part, le voyeurisme et le chant de la beauté du corps masculin chez Marie Nizet et Marguerite Burnat-Provins; d'autres, comme Anna de Noailles, Lucie Delarue-Mardrus, Hélène Vacaresco, et Gérard d'Houville, ont chanté le refuge dans la nature, l'enfance et l'exotisme, qui sont tous trois territoires sauvages hors de la société conventionnelle. Mais, de toutes ces amazones éprises de liberté, venant d'ailleurs et partant à la conquête de l'inconnu, il n'y a que Marie Krysinska qui ait su vraiment innover dans la forme poétique. Krysinska, pourtant fille d'un avocat de Varsovie, opta pour la vie de bohème, tandis que les autres étaient voyageuses et femmes du monde, et elles n'ont pas cherché le "dérèglement" de la forme, pour extrapoler sur l'expression de Rimbaud. L'attachement à des formes classiques dans la poésie féminine du début du siècle a donc aussi été un effet du conditionnement social, ces femmes adaptant leur imagination à une structure bien définie. Elles ressentaient le désir de se lancer dans l'infini tout en restant attachées par un fil à la tradition, un désir de révolution tempéré par une volonté d'ordre dans le monde, dictée par une adhésion à l'image conventionnelle de la femme du monde. 
Marie Krysinska, elle, errait dans les cabarets littéraires et artistiques parisiens à dominance masculine, à la même époque où Isabelle Eberhardt, une autre femme écrivain marginale et non conventionnelle, abandonnait la civilisation occidentale pour errer dans le désert saharien, et toutes deux se sont libérées de traditions littéraires. Au nomadisme, non seulement géographique, mais aussi social, est donc attaché un langage au pouvoir subversif de la société bourgeoise sédentaire. L'écriture cherchant à déstabiliser le territoire stable de la société conventionnelle, devient ellemême territoire instable car elle reproduit l'errance, chez Eberhardt avec des personnages vagabonds, libérés de toutes possessions, et chez Krysinska avec les danses, rondes et chansons. Les rondes de Krysinska représentent le mouvement perpétuel, et donc le lieu de l'écriture, que l'on espérait stable, est aussitôt déstabilisé. Les chansons représentent un genre hybride, et donc l'espace de l'écriture, que l'on espérait uni, est aussitôt désuni. Ce mouvement continuel est aussi celui de la fugue en musique, dont le thème et ses variantes s'échappent et se poursuivent les uns les autres. Entre vers et prose, Marie Krysinska fuguait sans cesse, et était, selon l'expression de Marguerite Duras, "totalement révolutionnaire" ou "totalement d'avant-garde" (61).

Car c'est aussi en larguant les amarres que peut naître le "nouveau," selon l'expression de Baudelaire ("Le Voyage"), et que l'on peut s'envoler vers l'Idéal, que la dernière strophe de "Devant le miroir" semble suggérer. La rencontre fugitive avec le passé permet une autre transformation, un saut dans une autre dimension, qui est celle des rêves, avec le verbe "songe," à double sens, celle de l'imagination, manifeste dans le monde de l'enfance, celle du mystère des choses cachées qu'essayaient de suggérer les Symbolistes. Cette réalité cachée est aussi une forme d'exil du poète symboliste par rapport à la réalité ordinaire, et ces efforts pour créer un langage qui évoquerait l'envers des choses, selon Mallarmé, "le sens mystérieux des aspects de l'existence" (La Vogue, n. pag.), conduiront au surréalisme tentant de traduire les images provenant du subconscient.

"Cet autre miroir" est "enchanté" comme cette autre réalité est magique. Le poète arrive donc à passer de l'autre côté du miroir, et par la magie de la poésie, le "on" se tranforme en "nous" de "nos jeunes cœurs," auquel le lecteur est convié à s'associer pour une expérience spirituelle universelle. C'est finalement le rêve ("songe") ou l'imagination (l'enfance de "nos jeunes cours"), la méditation, l'expérience spirituelle ou la rencontre avec l'Idéal ("ciel"), qui rendent les choses fertiles, qui font pousser des "herbes et des fleurs" là où elles ne pourraient pas survivre normalement, dans l'eau de l'étang, ou dans le ciel. Cette image de fertilité magique demande 
la suspension provisoire de l'incrédulité du lecteur, et renvoie à la fertilité espérée au début du poème, au commencement de l'aventure de l'écriture. De même, le lecteur, lorsqu'il lit un poème, pénètre dans un monde magique. Pour Krysinska, fixer son identité paraissait problématique, et c'est dans l'Idéal qu'elle s'échappait pour, peut-être, mieux se retrouver, fidèle à qui elle était, c'est-à-dire changeante, indéfinie et libre, la constance dans l'inconstance, baroque.

Il y a cependant un risque à pénétrer dans cette autre réalité, c'est celui de l'attirance de "l'eau de l'étang" pour l'enfant qui peut s'y noyer, symbole de la fascination de la mort. Le miroir derrière lequel on passe pour accéder à une autre réalité est symbole du passage de la vie à la mort. Le moi doit mourir pour que l'âme se libère. Le corps a disparu, l'image de soi, disloquée ou dépersonnalisée, a disparu, l'enfance a disparu. Le moi est pulvérisé dans l'expérience méditative, tout comme il est dissolu dans l'orgasme. Pour Krysinska, c'est en se fondant avec les choses, mais aussi en se perdant en elles, que le poète peut faire l'expérience de l'unité retrouvée, que l'Idéal d'harmonie peut être entrevu, ne serait-ce que le temps d'une "illumination," encore selon Rimbaud, ou d'une impression. Cet Idéal est figuré par l'union du ciel et de la terre des deux derniers vers ("Un morceau de ciel tombé / Où poussaient aussi des herbes et des fleurs"), du monde spirituel et du monde matériel, du monde des rêves et du monde tangible, du temple des arts, situé dans le "ciel," et de notions plus prosaïques comme des "herbes et des fleurs," dans un mouvement réciproque de l'un vers l'autre ("tomber" et "pousser"), mouvement qui crée paradoxalement l'équilibre entre deux mondes opposés, l'un étant le miroir de l'autre, œuvre d'un subtil métissage.

Mais, comme tout est mouvement, cet Idéal est aussi fugitif qu'une impression, et les trois derniers vers du poème font penser à une peinture impressionniste, sentiment renforcé par le mot "impressionnant," tandis que le miroir tremblant de l'eau de l'étang rappelle le flou artistique. Le tableau impressionniste produit le même effet sur les sens: la vue des images est floue, non limitée au contour des choses, les impressions se mélangent pour éveiller des sensations et hallucinations diverses. Le sujet est anihilé, ou s'endort, au profit des visions et des choses rêvées. Il semblerait donc qu'une vision "impressionniste" du monde, dans laquelle les couleurs se fondent et les différences ne sont pas aussi définies qu'on le croyait, réclame la disparition de l'individu, ou du moins de l'individualisme. Un monde où il n'y a pas de vérité absolue, où le mystère qui entoure les choses et les personnes leur donne justement leur valeur, où les sensations et les arts se mêlent, où la vision globale est encouragée plutôt que le profit individuel, où l'inconnu ne fait plus peur, est un monde 
fondé sur l'acceptance de l'autre et la fraternité entre les peuples. Dans un monde "impressionniste," ou de vision globale, les distinctions et hiérarchies entre blanc et noir ne sont plus aussi définitives et tenues pour vraies. Le métissage culturel représente donc l'espoir d'éliminer le racisme.

Une vision impressionniste pourrait être celle issue de la position ambiguë de l'immigrant, entre deux cultures. De ces zones "déterritorialisées," selon la formule de Gilles Deleuze et Félix Guattari, de ces "contact zones" de Mary Louise Pratt, ou encore de ces "border zones" de Renato Rosaldo, naissent des œuvres aux caractéristiques de métissages. Ces métissages sont subversifs à la fois du langage dominant de la culture d'accueil et du langage d'origine, comme l'a montré Samia Mehrez dans son étude de l'écrivain Beur (25-42): la deuxième génération d'immigrants nord-africains en France parle le verlan, où les syllabes des mots français sont inversées (comme "beur" pour "arabe"), et ce nouveau langage les distingue à la fois de leurs parents et des Français. De même, le nouveau langage de Krysinska est caractérisé par une nouvelle forme, entre vers et prose, un vers libre métissé-encore une fois, le troisième côté du triangle-, qui ne peut être catégorisé, et qui est à la fois un refus du polonais des parents (puisqu'elle utilise le français), et une subversion de la forme littéraire française classique. Krysinska a revendiqué à la fois la différence et l'acceptation, position de l'immigrant, au regard à la fois extérieur et intérieur. Dans son œuvre, l'ambiguïté entre vers et prose est accompagnée d'un métissage entre l'oralité et l'écriture, d'un mélange de tons, et d'un tissage entre la langue parlée et la langue poétique, comme nous l'avons vu dans "Devant le miroir," et qui aboutissent à la chanson de cabaret, genre hybride dont l'importance subversive et la popularité dans la culture française sont considérables.

Ces métissages de langues encouragent un dialogue où les différences sont mieux acceptées, ce qui est une résistance aux nationalisme, totalitarisme, et racisme. De plus, ils sont une réflexion de la quête du chant original, de l'espoir d'une renaissance de l'homme entier, morcelé entre corps et esprit, comme l'a montré Jacques Derrida, depuis Platon et ensuite Descartes. Le Symbolisme cherchait à faire une littérature synthétique: la poésie unirait tous les arts, la peinture (qui correspond au niveau graphique, visuel, ou sensuel), la musique (qui s'adresse directement aux émotions), et les mots (qui véhiculent des idées). En présentant la vie totale d'une âme, les Symbolistes cherchaient à retrouver l'harmonie du corps, des émotions et de la pensée. C'est dans ce contexte d'harmonie triangulaire, que la patrie recherchée est l'Idéal, une réalité spirituelle exempte de divisions entre les êtres, mais aussi à l'intérieur de l'être. C'est donc l'exil, ou le déracinement, qui non seulement promeut l'espoir d'une 
fraternité entre les peuples par le métissage des langues dans les poches de culture déterritorialisées, mais aussi qui entraîne vers un sentiment d'unité retrouvée au sein de l'être lui-même.

Flottant entre deux cultures, ou deux réalités, Marie Krysinska se permit de naviguer et de se renouveler sans cesse dans l'écriture, loin d'un carcan d'une définition trop rigide d'elle-même. Elle a recherché cette unité entre le corps, les émotions et l'intellect, et elle a participé symboliquement à l'unité entre les peuples par ses métissages de langues. Pour accéder à cette double unité, le moi doit disparaître, ou l'image du moi, ce qui vient peut-être plus naturellement à l'immigrée, puisque son image est déjà déstabilisée par l'exil. En poésie, l'image est floue, puis elle disparaît pour devenir symbole. L'ultime patrie, pour Krysinska comme pour les Symbolistes, ce fut donc l'Idéal, lieu doublement unificateur, où les notions de temps et d'espace, de stabilité, de définition et de moi n'ont plus cours, et qui est aussi symbole de liberté.

University of New Mexico 


\section{Works Cited}

Barre, André. Le Symbolisme. New York: Burt Franklin, 1911.

Deleuze, Gilles, and Félix Guattari. Pour une littérature mineure. Trans.

Dana Polan. Minneapolis: University of Minnesota Press, 1986.

Duras, Marguerite, and Xavière Gauthier. Les Parleuses. Paris: Les Editions de Minuit, 1985.

Godfrey, Sima. "Haute couture, haute culture." De la littérature française.

Ed. Denis Hollier, et al. Paris: Bordas, 1993. 715-23.

Krysinska, Marie. Intermèdes. Paris: Messein, 1904.

Krysinska, Marie. Joies Errantes. Paris: Lemerre, 1894.

Mallarmé, Stéphane. No title. La Vogue 18 Apr. 1886.

Mehrez, Samia. "Azouz Begag: Un di Zafas di Bidoufile (Azouz Begag: un des enfants du bidonville), or the Beur Writer: A Question of Territory." Yale French Studies 82 (1993): 25-42.

Michaud, Guy. Le Message poétique du Symbolisme. Paris: n.p., 1947.

Noailles, Anna de. Le Cour innombrable. Paris: Calman-Lévy, 1901.

Pratt, Mary Louise. "Criticism in the Contact Zone." Critical Theory, Cultural Politics, and Latin American Narrative. Ed. M. Bell, Albert H. Le May, and Leonard Orr. South Bend: University of Notre Dame Press, 1993.

Rosaldo, Renato. "Ideology, Place, and People Without Culture." Cultural Anthropology 3 (1988): 77-87.

Stamelman, Richard. "The Strangeness of the Other and the Otherness of the Stranger." Yale French Studies 82 (1993): 118-9. 
